\title{
Comparison of redox parameters in ovarian endometrioma and its malignant transformation
}

\author{
YOSHIKATSU FUJIMOTO ${ }^{1,2}$, SHOGO IMANAKA ${ }^{2,3}$, YUKI YAMADA $^{2}$, KENJI OGAWA $^{2}$, \\ FUMINORI ITO $^{4}$, NAOKI KAWAHARA ${ }^{2}$, CHIHARU YOSHIMOTO ${ }^{2}$ and HIROSHI KOBAYASHI ${ }^{2}$ \\ ${ }^{1}$ Department of Obstetrics and Gynecology, Japan Community Health Care Organization Yamato Koriyama, Yamato \\ Koriyama, Nara 634-1013; ${ }^{2}$ Department of Obstetrics and Gynecology, Nara Medical University, Kashihara, \\ Nara 634-8522; ${ }^{3}$ IVF Osaka Clinic, Osaka 577-0012; ${ }^{4}$ Department of Obstetrics and Gynecology, \\ Nara Prefecture General Medical Center, Nara 631-0846, Japan
}

Received March 27, 2018; Accepted July 25, 2018

DOI: $10.3892 / \mathrm{ol} .2018 .9242$

\begin{abstract}
The present study aimed to evaluate the levels of oxidative stress and antioxidant markers in benign endometrioma (OE) and its malignant transformation [endometriosis-associated ovarian cancer (EAOC)] by measuring 8-hydroxy-2-deoxyguanosine (8-OHdG), heme oxygenase-1 (HO-1) and total antioxidant capacity (TAC/Heme-iron) alterations associated with disease progression. Cyst fluid samples from 44 patients with OE and 14 patients with EAOC were studied using an enzyme-linked immunosorbent assay. A $\chi^{2}$ test, t-test and Pearson correlation test were performed using SPSS version 22.0. The cut-off point, sensitivity and specificity of each marker for EAOC diagnosis were evaluated by receiver operating characteristic curve analysis. Cyst fluid 8-OHdG and HO-1 levels in the EAOC group were significantly decreased compared with the OE subjects $(\mathrm{P}=0.013$ and $\mathrm{P}<0.001$, respectively). The levels of TAC/Heme-iron in patients with EAOC were significantly higher compared with those in the OE subjects $(\mathrm{P}<0.001)$. The present study demonstrated a positive correlation between 8-OHdG and HO-1 levels ( $\mathrm{P}=0.012)$. HO-1 exhibited the highest discriminant value for EAOC (Area Under the Curve=0.901). The optimal cut-off point of HO-1 for the diagnosis of EAOC was $2.314 \mathrm{ng} / \mathrm{ml}$, with a sensitivity and specifity of 95.2 and $85.7 \%$, respectively. The present study revealed a clear separation between the overall redox state in OE and EAOC. It was concluded that characteristic alterations in important factors in redox may be helpful for understanding the pathogenesis of the malignant transformation of endometriosis.
\end{abstract}

Correspondence to: Dr Hiroshi Kobayashi, Department of Obstetrics and Gynecology, Nara Medical University, 840 Shijo-cho, Kashihara, Nara 634-8522, Japan

E-mail: hirokoba@naramed-u.ac.jp

Key words: ovarian endometrioma, endometriosis-associated ovarian cancer, 8-hydroxy-2-deoxyguanosine, heme oxygenase-1, total antioxidant capacity

\section{Introduction}

Endometriosis is defined as the presence of functioning endometrial glands and stroma outside the uterus, most commonly in the pelvic peritoneum and ovaries. This disorder is a benign estrogen-dependent disease of reproductive age, although ovarian endometrioma (OE) increases the subsequent risk of developing endometriosis-associated ovarian cancer (EAOC) $(1,2)$. EAOC occurs in $0.72 \%$ of women with endometriosis in Japan (1). The risk factors for malignant transformation include older age ( $>45$ years old) and large tumor size ( $>7 \mathrm{~cm})(2)$. Ovarian cancer is an important cause of cancer morbidity and mortality, largely due to the late stage of presentation. The mechanisms involved in malignant transformations warrant further investigation.

Repeated bleeding episodes occur in endometriosis and result in a local accumulation of hemoglobin, heme and iron species (3), which leads to increased formation of reactive oxygen species (ROS) (4). Generally, ROS consist principally of molecules including the superoxide anion $\left(\mathrm{O}_{2}{ }^{-}\right)$, hydroxyl radicals $(\bullet \mathrm{OH})$ and hydrogen peroxide $\left(\mathrm{H}_{2} \mathrm{O}_{2}\right)$. 8-hydroxy-2-deoxyguanosine $(8-\mathrm{OHdG})$ is considered to be a biomarker of ROS-mediated DNA damage, as guanosine is the most oxidized among the DNA nucleobases (5). Toxic heme also induces a stress-responsive enzyme, heme oxygenase-1 (HO-1), that is responsible for the degradation of heme to carbon monoxide (CO), bilirubin and iron (6). HO-1 and their products exert beneficial effects through protection against oxidative injury. In response to an excess of ROS, macrophages secrete antioxidants, and regulate redox signaling and the inflammatory microenvironment, via control of the expression of a transcription factor, NRF2 (Nuclear factor erythroid-derived factor 2-related factor 2), during endometriosis regeneration (3). NRF2 also regulates the expression of HO-1 and antioxidants. HO-1 is thought to be an oxidative stress marker $(6,7)$. On the other hand, the combined activity of all antioxidants is measured via the total antioxidant capacity (TAC), instead of measuring the activity of each agent separately, for example superoxide dismutase (SOD), catalase (CAT) and glutathione peroxidase (GSH-Px) (8). 
TAC/Heme-iron reflects the antioxidant capacity of water-soluble molecules, since the majority of iron exists as heme-iron in OE and EAOC cyst fluid samples (9).

Oxidative stress may be involved in the progression of the malignant transformation of endometriosis $(10,11)$. However, no reports on the oxidant-antioxidant profile in the cyst fluid of patients suffering from OE and EAOC exist, to the best of our knowledge. The aim of this study was to analyze redox biomarkers, pro-oxidants (the in vivo DNA damage marker 8-OHdG, and the oxidative stress marker HO-1) and antioxidants (TAC/Heme-iron) in the cyst fluids of patients with benign $\mathrm{OE}$ and already-growing EAOC.

\section{Materials and methods}

Patient selection. The present study was conducted on 14 histopathologically confirmed cases of EAOC and the 44 cases of benign OE. To avoid selection bias in the EAOC cases, morphological documentation of the continuous transition from benign endometriotic epithelial cells to atypical endometriosis, and finally to invasive carcinoma, was confirmed within the same specimen. A total of 58 cyst fluid sample specimens were collected from the Department of Gynecology at Nara Medical University Hospital, Japan, between January 2006 and December 2012. Specific exclusion criteria considered for the present study were acute or chronic diseases, including diabetes, immune dysfunction or any other malignancy. None of the study subjects were under antioxidant supplementation, hormonal therapy or chemotherapy prior to the surgery. The data on patient demographic features and clinicopathological characteristics were collected from a database containing comprehensive medical records and pathology reports. The protocols were approved by The Ethics Committee of Nara Medical University (reference no. 2012-541). Written informed consent was obtained from each study subject, and all subjects consented to donate cyst fluid samples.

Analytical methods. Cyst fluid samples were collected at the time of surgery. Following centrifugation at 3,000 x g for $15 \mathrm{~min}$, specimens were immediately aliquoted and frozen at $-70^{\circ} \mathrm{C}$ within $1 \mathrm{~h}$ of collection. A histological diagnosis was confirmed via surgical pathology.

Estimation of 8-OHdG and $\mathrm{HO}-1$. Cyst fluid samples were used for the measurement of 8-hydroxy-2-deoxy guanosine $(8-\mathrm{OHdG})$ levels using a competitive in vitro ELISA kit (Catalog\# KOG-HS10/EC, NIKKEN SEIL Co., Ltd, Shizuoka, Japan), and HO-1 levels using StressXpress ${ }^{\circledR} \mathrm{HO}-1$ ELISA kits (Catalog\# SKT-111-96, StressMarq Biosciences, Inc., Victoria, BC, Canada). Dilution linearity and parallelism, in addition to intra- and inter-assay variability, were assessed using in-house calibrators. The two ELISA kits exhibited a linear response in the range of $\geq 3$ orders of magnitude. The assay variances of all methods described above were $<10 \%$.

Assay of total antioxidant status. Total antioxidant capacity (TAC) in the cyst fluid was measured using the TAC Assay kit (Metallogenics Co., Ltd., Chiba, Japan), which uses a copper (II) reduction assay with bathocuproinedisulfonic acid disodium salt as the chelating agent (the CUPRAC-BCS assay). This assay was applied to measure the antioxidants as reductants in a redox-linked colorimetric procedure using a spectrophotometer (Bio Aquarius; Cecil Instruments Ltd., Cambridge, UK), as described previously $(12,13)$.

Measurement of heme iron concentration. Cyst fluids were added to 96-well plates and alkalized with $\mathrm{NaOH}$ to adjust the $\mathrm{pH}$ to $>10$. The resultant solution was subjected to heme measurement using the Metalloassay LS Heme Assay kit (Metallogenics Co., Ltd.), based on the Triton-methanol colorimetric assay (14). The optical density at $400 \mathrm{~nm}$ was determined using a microtiter plate reader.

Statistical analysis. All values are expressed as the mean \pm standard deviation. Differences between the groups of patients were estimated by Mann-Whitney U test. Categorical variables are presented as absolute numbers (frequency percentages) and analyzed via the $\chi^{2}$ test. Correlations between levels of oxidative stress biomarkers and antioxidant status were evaluated using Pearson's correlation coefficient. Receiver Operative Characterisitic (ROC) curve analysis was used to identify the best discriminating threshold of the cyst fluid iron levels for differential diagnosis between EAOC and OE. Analyses were performed using SPSS (v.22.0; IBM Corp., Armonk, NY, USA). $\mathrm{P}<0.05$ was considered to indicate a statistically significant difference.

\section{Results}

Baseline characteristics. The principal clinical and pathological characteristics of the subjects with benign $\mathrm{OE}$ and patients with EAOC are presented in Table I. Age $(\mathrm{P}=0.001)$, premenopausal status $(\mathrm{P}=0.001)$ and the maximum diameter of the cyst $(\mathrm{P}=0.006)$ differed significantly between the two groups. There were no statistical differences in parity and serum CA125 levels between the two groups $(\mathrm{P}>0.05)$.

Cyst fluid marker levels in patients with OE and EAOC. As presented in Fig. 1, box and whisker plots represent the median levels and the interquartile range (box) of $8-\mathrm{OHdG}$ (Fig. 1A), HO-1 (Fig. 1B) and TAC/Heme-iron (Fig. 1C) for each studied group. 8-OHdG concentrations in all samples ranged between 0.164 and $49.82 \mathrm{ng} / \mathrm{ml}$. The concentrations were highly divergent between the patients, with a few having extremely high concentrations and a few with very low concentrations. Cyst fluid 8-OHdG levels were significantly lower in patients with EAOC compared with $\mathrm{OE}$ $(\mathrm{P}=0.013$; Fig. 1A). EAOC patients had significantly lower HO-1 levels compared with subjects with $\mathrm{OE}(\mathrm{P}<0.001$; Fig. 1B). Compared with OE, TAC/Heme-iron levels were significantly higher in EAOC $(\mathrm{P}<0.001$; Fig. 1C). The marker values by each type of cyst are presented in Table II. Diminished oxidative damage in the malignant group compared with the benign group was accompanied by the increase in antioxidant protection.

Correlation between patient ages and cyst fluid marker levels. Since the EAOC group was significantly older compared with the OE group $(\mathrm{P}=0.001)$, the correlation between age and cyst fluid marker levels was analyzed. No significant correlation 
Table I. Clinical and tumor characteristics of the study population.

\begin{tabular}{|c|c|c|c|}
\hline $\begin{array}{l}\text { Baseline characteristics } \\
\text { of two groups }\end{array}$ & $\begin{array}{c}\text { Benign } \\
\text { endometrioma group }\end{array}$ & $\begin{array}{l}\text { Endometriosis-associated } \\
\text { ovarian cancer group }\end{array}$ & P-value \\
\hline No. & 44 & 14 & \\
\hline \multicolumn{4}{|l|}{ Age at diagnosis, } \\
\hline Mean \pm SD & $36.9 \pm 8.4$ & $50.0 \pm 13.2$ & \multirow[t]{2}{*}{0.001} \\
\hline Median (range) & $38.0(21-62)$ & $44.5(36-78)$ & \\
\hline Nulliparous n (\%) & $19(43.2 \%)$ & $7(50.0 \%)$ & 0.665 \\
\hline Premenopause n (\%) & $42(95.5 \%)$ & $9(64.2 \%)$ & 0.007 \\
\hline \multicolumn{4}{|l|}{ Tumor size $(\mathrm{cm})^{\mathrm{a}}$} \\
\hline Mean \pm SD & $7.12 \pm 2.78$ & $12.0 \pm 6.43$ & \multirow[t]{2}{*}{0.006} \\
\hline Median (range) & $6.8(2.7-13.6)$ & $10.6(4.2-25.5)$ & \\
\hline \multicolumn{4}{|l|}{ CA125 } \\
\hline Mean \pm SD & $92.1 \pm 70.3$ & $375.2 \pm 658.0$ & \multirow{10}{*}{0.531} \\
\hline Median (range) & $66.5(10-316)$ & $47.0(7.0-2141)$ & \\
\hline \multirow[t]{5}{*}{ Pathology } & \multirow[t]{5}{*}{ Endometriosis } & Clear cell $(n=6)$ & \\
\hline & & Endometrioid $(\mathrm{n}=1)$ & \\
\hline & & Serous $(n=3)$ & \\
\hline & & Mucinous $(\mathrm{n}=1)$ & \\
\hline & & Other $(n=3)$ & \\
\hline \multirow{3}{*}{$\begin{array}{l}\text { The international federation of gynecology } \\
\text { and obstetrics stage }\end{array}$} & \multirow[t]{3}{*}{-} & IA (n=3) & \\
\hline & & $\mathrm{IC}(\mathrm{n}=10)$ & \\
\hline & & $\operatorname{IIIC~(n=1)~}$ & \\
\hline
\end{tabular}

${ }^{a}$ Maximum diameter of tumors. SD, standard deviation; CA125, cancer antigen 125.
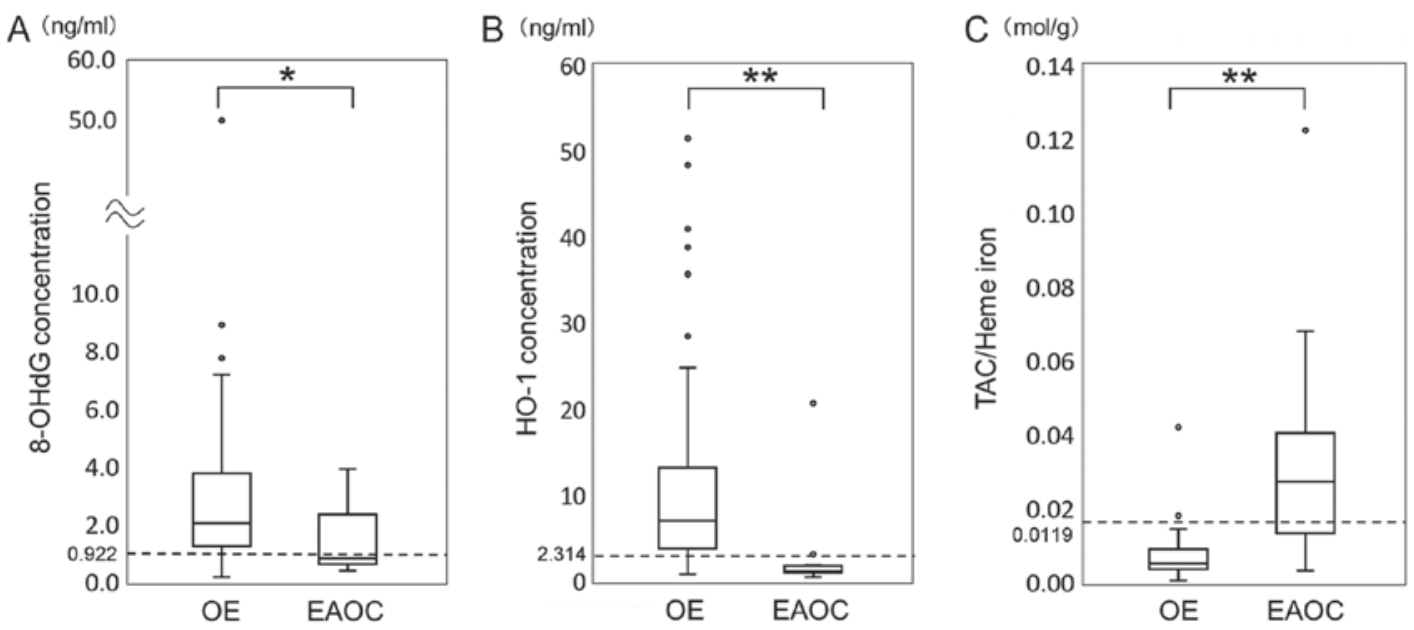

Figure 1. Cyst fluid marker levels in patients with OE and EAOC. The distribution of marker levels is presented for each studied group. Cyst fluid levels were studied in patients with OE ( $\mathrm{n}=44$ ) and EAOC (n=14). (A) 8-OHdG; (B) HO-1; and (C) TAC/Heme-iron. The dashed horizontal line represents the cut-off level for each marker. ${ }^{*} \mathrm{P}=0.013 ;{ }^{* *} \mathrm{P}<0.001$.

was identified between patient age and the cyst fluid levels of 8-OHdG, HO-1 or TAC/Heme-iron (Fig. 2).

Correlation between tumor size and cyst fluid marker levels. The present study subsequently analyzed the correlation between cyst fluid marker levels and tumor size. No significant differences were observed between 8 -OHdG (Fig. 3A) and HO-1 levels (Fig. 3B) and tumor size. Cyst fluid
TAC/Heme-iron levels exhibited a positive association with tumor size (Fig. 3C).

Correlation between cyst fluid 8-OHdG levels, $\mathrm{HO}-1$ levels and TAC/Heme-iron levels. With the use of Pearson's correlation coefficient analysis, $8-\mathrm{OHdG}$ levels were demonstrated to be positively correlated with HO-1 levels ( $\mathrm{P}=0.012$; Fig. $4 \mathrm{~A})$. The correlation between the antioxidant marker TAC/Heme-iron, 
Table II. Level of three markers in benign OE and EAOC.

\begin{tabular}{lccr}
\hline & \multicolumn{2}{c}{ Cyst fluid value } & \\
\cline { 2 - 3 } Parameter & OE group & EAOC group & P-value \\
\hline 8-OHdG (ng/ml) & $2.023(0.16-49.98)$ & $0.820(0.39-3.89)$ & $0.013^{\mathrm{a}}$ \\
HO-1 (ng/ml) & $7.00(0.83-51.47)$ & $1.15(0.42-20.69)$ & $<0.001^{\mathrm{a}}$ \\
TAC/Heme-iron (mol/g) & $0.0051(0.0005-0.0420)$ & $0.0270(0.0030-0.1226)$ & $<0.001^{\mathrm{a}}$ \\
\hline
\end{tabular}

Data are presented as the median (range). Statistical analysis was performed using the Mann-Whitney U test. ${ }^{a} \mathrm{P}<0.05$ was considered statistically significant. 8-OHdG, 8-hydroxy-2-deoxy guanosine; HO-1, heme oxygenase-1; TAC, total antioxidant capacity; OE, benign endometrioma; EAOC, endometriosis-associated ovarian cancer.
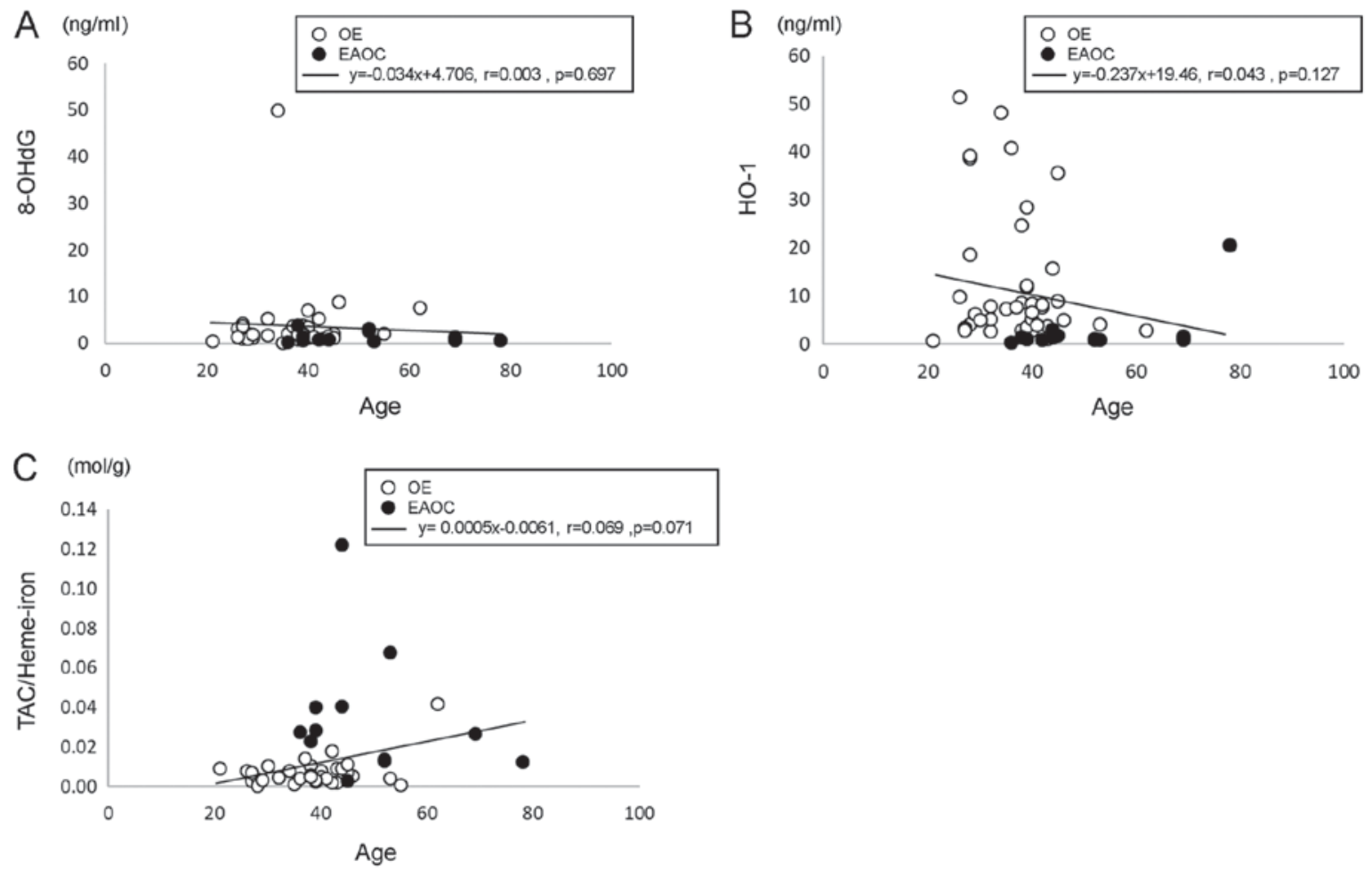

Figure 2. Correlation between patient ages and cyst fluid marker levels. Correlation between (A) cyst fluid 8-OHdG levels, (B) HO-1 levels and (C) TAC/Heme-iron levels and patient age at surgery. The open circle represents the individual value of an endometriosis subject. The closed circle represents the individual value of an EAOC subject.

and the levels of the oxidative stress markers 8 -OHdG and HO-1, was assessed. No correlation existed between TAC/Heme-iron and 8-OHdG levels (Fig. 4B), or between TAC/Heme-iron and HO-1 levels (Fig. 4C).

Evaluation of cyst fluid marker levels as potential biomarkers for differential diagnosis between EAOC and OE. Following quantitative measurement of each marker in 58 cyst fluid samples, ROC curves were applied to assess the potential utility of cyst fluid levels in diagnosing EAOC from OE (Fig. 5). The diagnostic sensitivity and specificity of $8-\mathrm{OHdG}, \mathrm{HO}-1$ and $\mathrm{TAC} / \mathrm{Heme}$-iron were analyzed and the results are presented in Fig. 5. The summary ROC curve illustrated that the AUC was 0.741 based on cyst fluid 8-OHdG levels (Fig. 5A). The detection sensitivity and specificity of $8-\mathrm{OHdG}$ were 91.7 and $58.3 \%$, respectively. HO-1 exhibited a higher detection sensitivity (95.2\%) and specificity (85.7\%). The corresponding AUC and cut-off point were 0.901 and 2.314, respectively. The ROC curves demonstrated an optimum cut-off point of 0.0119 (for TAC/Heme-iron) to distinguish EAOC from OE, yielding 91.7\% sensitivity and $91.7 \%$ specificity. The corresponding AUC was 0.898. As presented in Fig. 1, the dashed horizontal line represents the cut-off level for each marker. Among the indicators investigated, HO-1 and Tac/Heme-iron exhibited the highest discriminant value for EAOC (Fig. 5B and C).

\section{Discussion}

This study evaluated the cyst fluid levels of oxidative stress markers (8-OHdG and HO-1), and antioxidant capacity (TAC/Heme-iron), in patients with benign $\mathrm{OE}$ and already-growing EAOC. Lower pro-oxidant (8-OHdG and 

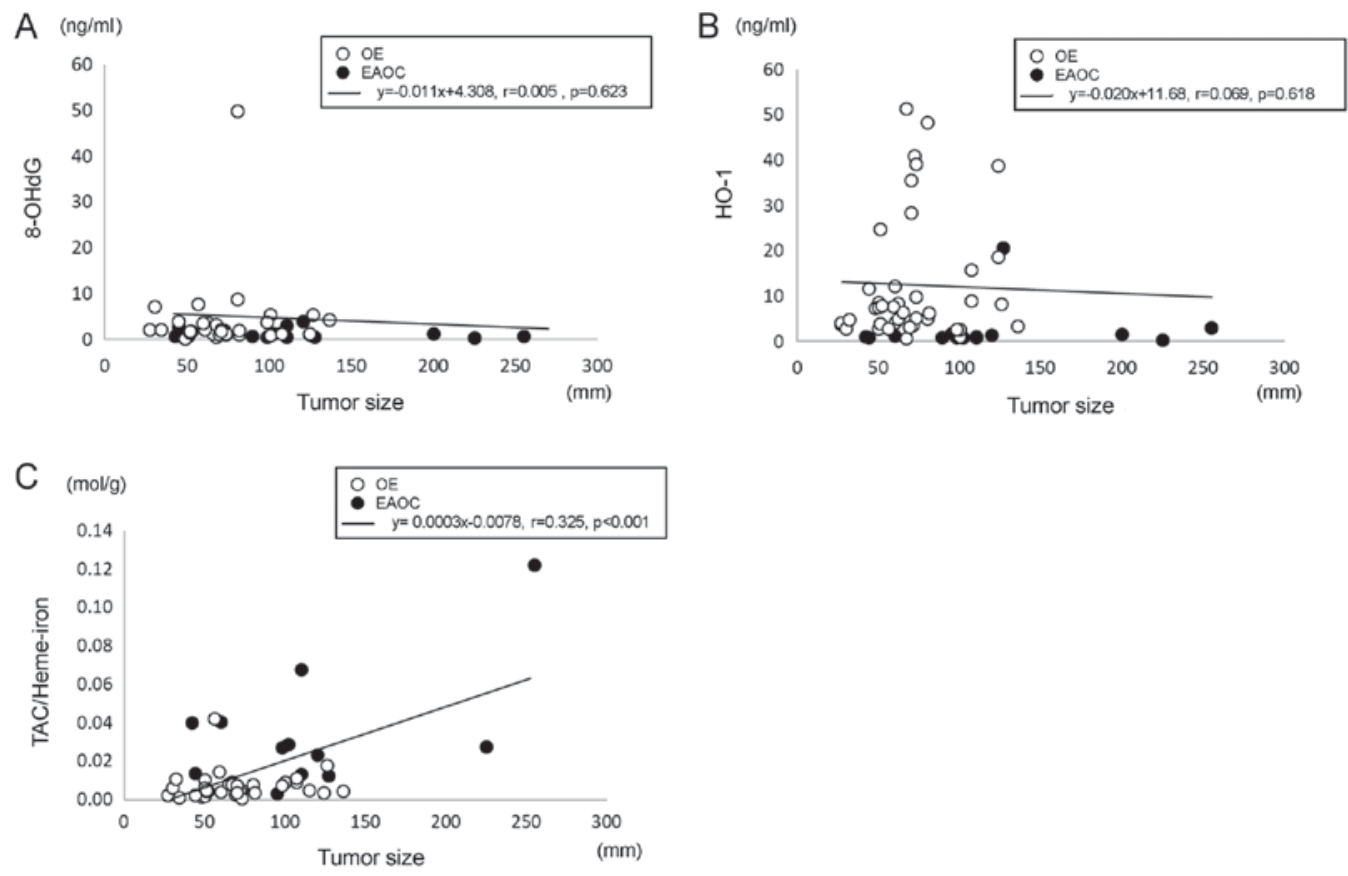

Figure 3. Correlation between tumor size and cyst fluid marker levels. Correlation between cyst fluid 8-OHdG levels (A), HO-1 levels (B) and TAC/Heme-iron levels (C) and tumor size. The open circle represents the individual value of an endometriosis subject. The closed circle represents the individual value of an EAOC subject.
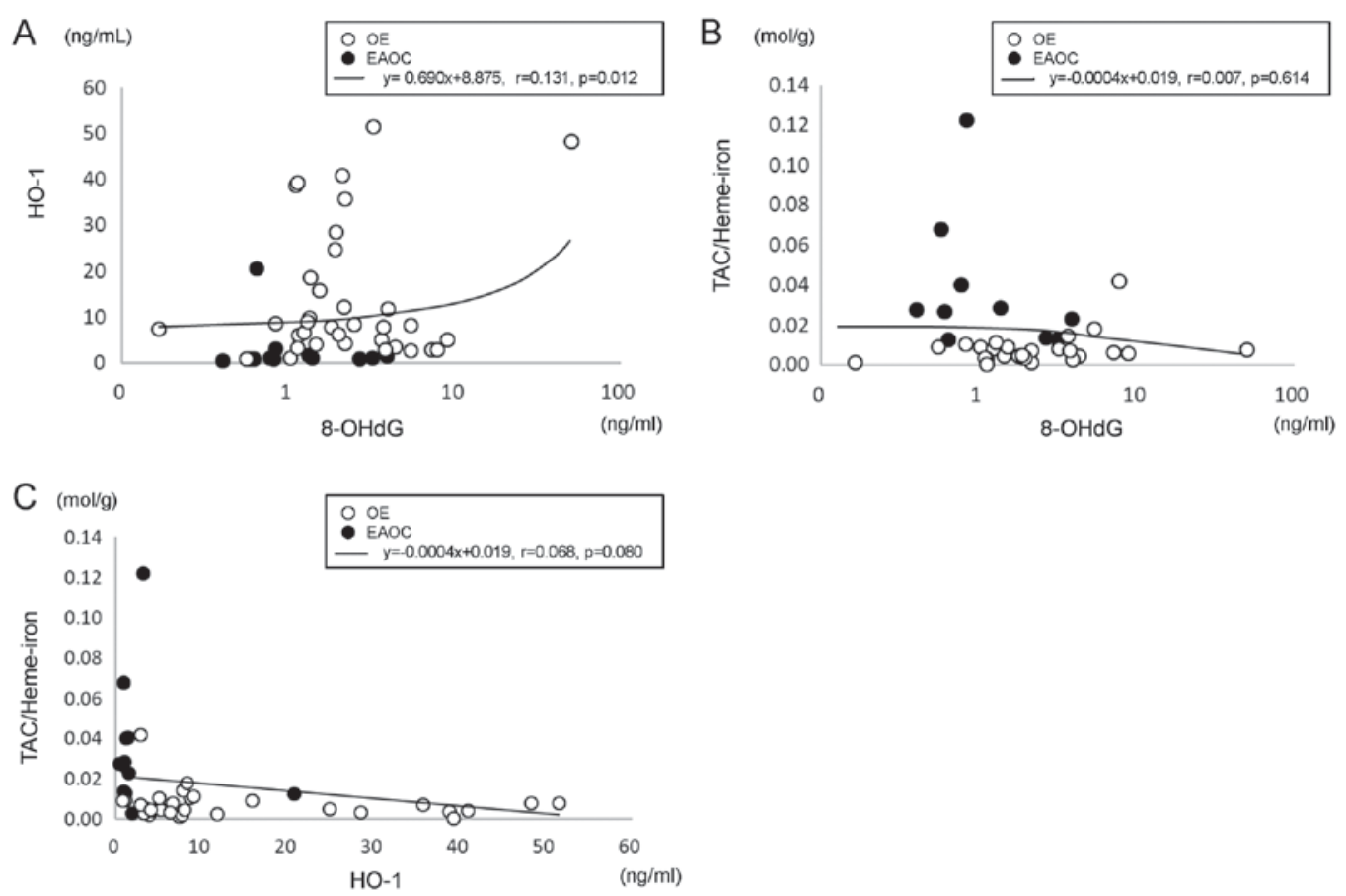

Figure 4. Correlation between cyst fluid 8-OHdG levels, HO-1 levels and TAC/Heme-iron levels. Correlation analysis between (A) 8-OHdG and HO-1, (B) 8-OHdG and TAC/Heme-iron and (C) HO-1 and TAC/Heme-iron.

HO-1) levels were observed in the cyst fluid of the EAOC group in comparison with the benign OE group. By contrast, the cyst fluid anti-oxidant TAC/Heme-iron was upregulated in the EAOC group compared with the OE group. HO-1 exhibited the highest discriminant value for differentiating between benign and malignant cyst fluids. The present study revealed a clear separation of the overall redox state between OE and EAOC. Therefore, redox imbalance may be implicated in the etiology of the malignant transformation of endometriosis.

Firstly, there is increasing evidence that oxidative stress is one of the key factors in the establishment and progression of endometriosis (15-17). High levels of oxidized DNA adduct 8-OHdG were observed in the cyst fluid from OE cases, suggesting increased oxidative stress and frequent DNA mutations (18). HO-1 may be expressed in endometriosis, particularly in red lesions (19), and 
A

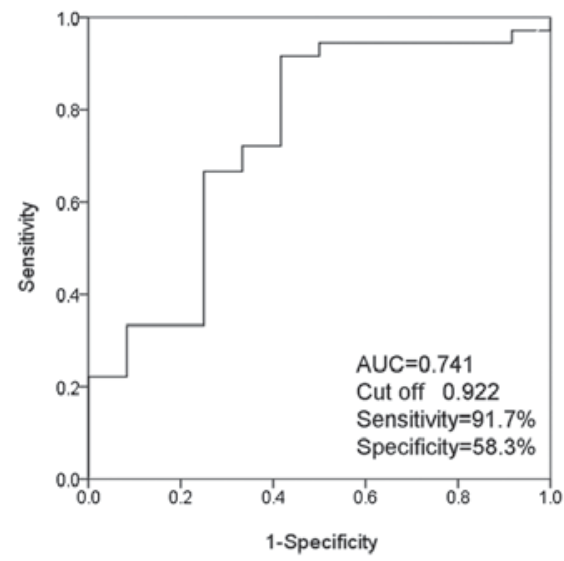

B

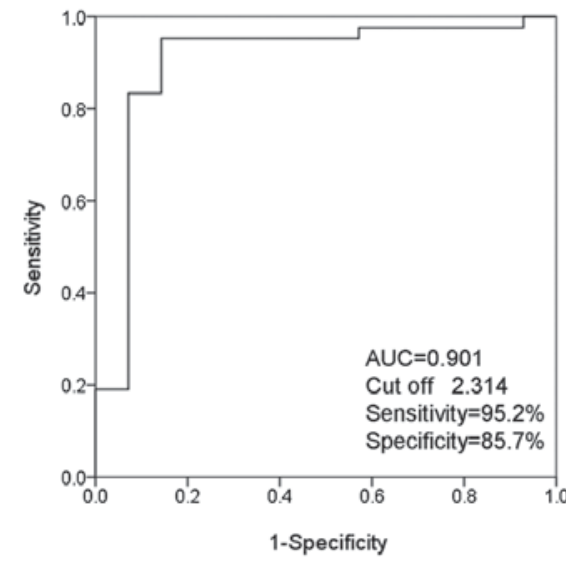

C ROC curves TAC/Heme-iron

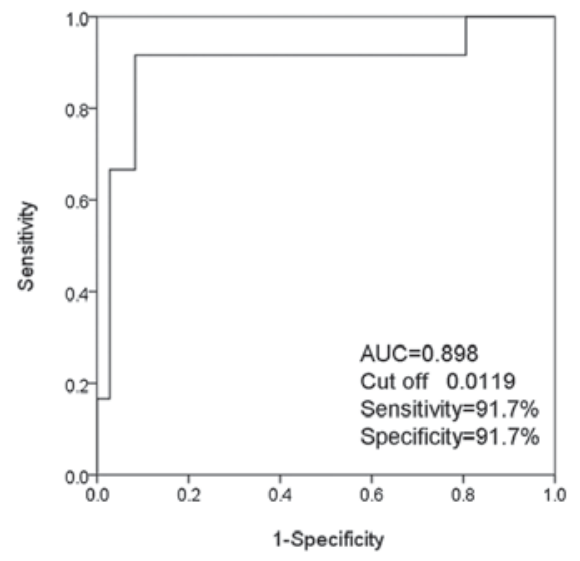

Figure 5. ROC plots for the predictions of EAOC via indicators. Receiver-operating characteristic analysis demonstrated a higher area under the curve for the ratio of the pixel density value of (A) 8-OHdG, (B) HO-1 and (C) TAC/Heme-iron.

also in macrophages accumulated in endometriotic tissues (20). A number of studies have confirmed a compromised antioxidant capacity in endometriosis $(4,10,12,21,22)$. Total antioxidant activity scavenges excess intracellular free radicals and augments cellular antioxidant defenses. These findings support the idea that higher oxidative stress-induced DNA damage and mutations, and lower antioxidant capacity, are associated with endometriosis progression, possibly directing it towards a pre-malignant phenotype (17). HO-1 may terminate the further expansion of pre-malignant endometriotic cells.

Secondly, few studies have examined the oxidant-antioxidant profile in EAOC. An immunohistochemical study demonstrated that EAOC tumor cells had weaker nuclear and cytoplasmic 8-OHdG expression compared with tumors adjacent to $\mathrm{OE}$ and benign endometriotic epithelial cells (23), suggesting an alleviation of oxidative stress during the process of malignant transformation. Contrary to previous reports (24), the present results indicated that reduced accumulation of ROS-mediated oxidative damage may predispose to cancer. In general, HO-1 influences tumor formation and progression, and its overexpression has been associated with tumor growth, aggressiveness, metastasis and angiogenetic potential, resistance to therapy, tumor escape and a poor prognosis $(7,25)$. In contrast to the well-known role of HO-1 in tumor aggressiveness, the present study suggests that diminished HO-1 expression may favor the progression from OE to EAOC. Meanwhile, HO-1 serves a key role in preventing tumor initiation and carcinogenesis $(25,26)$. Indeed, HO-1 expression was downregulated in early-stage lung carcinoma (27), suggesting an opposing role of $\mathrm{HO}-1$ in different neoplasms or at different times during tumor progression. Increased expression of HO-1 may protect benign $\mathrm{OE}$ against oxidative stress-induced injury; however, HO-1 has been suggested to be a negative factor contributing to tumor progression in EAOC. In other words, HO-1 is upregulated in pre-transformation endometriotic cells, although it remains at the basal level in already-growing cancer. Another possibility is that patients with EAOC had much lower levels of hemoglobin, heme and iron species compared with endometriotic cyst samples (9), supporting the idea that EAOC is associated with lower HO-1 levels compared with OE. Although the exact roles of HO-1 in malignant transformation remain controversial, HO-1 serves a dual (promoting or inhibitory) role at different stages of tumor initiation and progression $(7,27)$. The present study presents evidence supporting the idea that the pre-tumorigenic environment of endometriois induces a redox signaling switch in the oncogenic process.

Thirdly, an elevation in the TAC status in the malignant group suggested increased production of antioxidants in response to an enhanced level of oxidative damage. It was hypothesized that increased expression of TAC may promote the proliferation and survival of EAOC cells. The observed result was comparable to existing results reported in similar studies: Overexpression of a series of antioxidant genes counters cell and DNA damage originating from oxidative stress in liver, thyroid, breast, colon and pancreas cancer (28). Antioxidants alleviate cell death by scavenging surplus oxidative stress, thus allowing for survival. Taken together, enhancing antioxidant defenses and reducing oxidative stress may predispose to malignancy.

It has previously been established that increased intracellular ROS and decreased antioxidant molecules frequently initiate carcinogenesis in various types of cancer (24). The patients with EAOC exhibited an opposite pattern of altered expression of pro- and anti-oxidants. The present study highlighted certain evidence suggesting a redox change, which may drive the progression of already-growing cancer, although not the initiation of carcinogenesis. Therefore, characteristic alterations in redox parameters may depend on the investigation of different stages of tumor 'initiation' and 'progression'.

Finally, the present findings emphasize the requirement to identify risk factors associated with the malignant transformation of $\mathrm{OE}$ to predict it at the earliest stages. Among redox parameters, HO-1 may be a candidate marker to estimate the risk of malignant transformation. If there is a close association between serum and cyst fluid HO-1 levels, HO-1 has potential as a serum biomarker that may help to stratify the severity of $\mathrm{OE}$, to improve risk stratification for developing EAOC, and to accurately predict the prognosis of patients with EAOC. 
In conclusion, the present study revealed an imbalance in the redox system of the tumor microenvironment due to alterations in oxidative stress markers and antioxidant capacity between $\mathrm{OE}$ and EAOC. HO-1 may protect OE against oxidative stress-induced injury; however, in already-growing EAOC, excess antioxidants favor tumor progression. Further study of the molecular pathways involving redox balance may strengthen the understanding of how the local intracystic microenvironment initiates and sustains the transition of benign cells toward malignant forms. There is a gradient of redox expression and complexity in malignant transformation from benign $\mathrm{OE}$ to EAOC.

\section{Acknowledgements}

Not applicable.

\section{Funding}

The present study was supported by the Takeda Science Foundation (JSPS KAKENHI grant no. JP16K11150) and the Tohoku Bureau of Economy, Trade and Industry (grant no. Tohoku 1607028).

\section{Availability of data and materials}

All data generated or analysed during the present study are included in this published article.

\section{Authors' contributions}

SI, KO and NK collected patients samples. FI, YY and CY collected patients data and performed analyses. YF measured the samples and performed analyses. HK contributed to conception and design, acquisition of data, and analysis and interpretation of data. HK was also involved in drafting the manuscript or revising it critically for important intellectual content. All authors read and approved the final manuscript.

\section{Ethics approval and consent to participate}

The protocols were approved by The Ethics Committee of Nara Medical University (reference no. 2012-541). Written informed consent was obtained from each study subject, and all subjects consented to donate cyst fluid samples.

\section{Patient consent for publication}

Written informed consent was obtained from each study subject, and all subjects consented to donate cyst fluid samples.

\section{Competing interests}

The authors declare that they have no competing interests.

\section{References}

1. Kobayashi H, Sumimoto K, Moniwa N, Imai M, Takakura K, Kuromaki T, Morioka E, Arisawa K and Terao T: Risk of developing ovarian cancer among women with ovarian endometrioma: A cohort study in Shizuoka, Japan. Int J Gynecol Cancer 17: 37-43, 2007.
2. Kobayashi H: Ovarian cancer in endometriosis: Epidemiology, natural history, and clinical diagnosis. Int J Clin Oncol 14: 378-382, 2009.

3. Kobayashi H: Potential scenarios leading to ovarian cancer arising from endometriosis. Redox Rep 21: 119-126, 2016.

4. Iwabuchi T, Yoshimoto C, Shigetomi H and Kobayashi H: Oxidative stress and antioxidant defense in endometriosis and its malignant transformation. Oxid Med Cell Longev 2015: 848595, 2015.

5. Kasai H: Analysis of a form of oxidative DNA damage, 8-hydroxy-2'-deoxyguanosine, as a marker of cellular oxidative stress during carcinogenesis. Mutat Res 387: 147-163, 1997.

6. Ryter SW and Choi AM: Heme oxygenase-1: Redox regulation of a stress protein in lung and cell culture models. Antioxid Redox Signal 7: 80-91, 2005.

7. Nemeth Z, Li M, Csizmadia E, Döme B, Johansson M, Persson JL, Seth P, Otterbein L and Wegiel B: Heme oxygenase-1 in macrophages controls prostate cancer progression. Oncotarget 6: 33675-33688, 2015.

8. Zamani-Ahari U, Zamani-Ahari S, Fardi-Azar Z, Falsafi P and Ghanizadeh M: Comparison of total antioxidant capacity of saliva in women with gestational diabetes mellitus and non-diabetic pregnant women. J Clin Exp Dent 9: e1282-e1286, 2017.

9. Yoshimoto C, Iwabuchi T, Shigetomi H and Kobayashi H: Cyst fluid iron-related compounds as useful markers to distinguish malignant transformation from benign endometriotic cysts. Cancer Biomark 15: 493-499, 2015.

10. Scutiero G, Iannone P, Bernardi G, Bonaccorsi G, Spadaro S, Volta CA, Greco P and Nappi L: Oxidative stress and endometriosis: A systematic review of the literature. Oxid Med Cell Longev 2017: 7265238, 2017.

11. Worley MJ, Welch WR, Berkowitz RS and Ng SW: Endometriosis-associated ovarian cancer: A review of pathogenesis. Int J Mol Sci 14: 5367-5379, 2013.

12. Nasiri N, Moini A, Eftekhari-Yazdi P, Karimian L, Salman-Yazdi R and Arabipoor A: Oxidative stress statues in serum and follicular fluid of women with endometriosis. Cell J 18: 582-587, 2017.

13. Campos C, Guzmán R, López-Fernández E and Casado A: Evaluation of the copper(II) reduction assay using bathocuproinedisulfonic acid disodium salt for the total antioxidant capacity assessment: The CUPRAC-BCS assay. Anal Biochem 392: 37-44, 2009.

14. Pandey AV, Joshi SK, Tekwani BL and Chauhan VS: A colorimetric assay for heme in biological samples using 96-well plates. Anal Biochem 268: 159-161, 1999.

15. Di Emidio G, D'Alfonso A, Leocata P, Parisse V, Di Fonso A, Artini PG, Patacchiola F, Tatone C and Carta G: Increased levels of oxidative and carbonyl stress markers in normal ovarian cortex surrounding endometriotic cysts. Gynecol Endocrinol 30: 808-812, 2014.

16. Polak G, Wertel I, Barczyński B, Kwaśniewski W, Bednarek W and Kotarski J: Increased levels of oxidative stress markers in the peritoneal fluid of women with endometriosis. Eur J Obstet Gynecol Reprod Biol 168: 187-190, 2013.

17. Carvalho LF, Abrão MS, Biscotti C, Sharma R, Nutter B and Falcone T: Oxidative cell injury as a predictor of endometriosis progression. Reprod Sci 20: 688-698, 2013.

18. Yamaguchi K, Mandai M, Toyokuni S, Hamanishi J, Higuchi T, Takakura K and Fujii S: Contents of endometriotic cysts, especially the high concentration of free iron, are a possible cause of carcinogenesis in the cysts through the iron-induced persistent oxidative stress. Clin Cancer Res 14: 32-40, 2008.

19. Van Langendonckt A, Casanas-Roux F, Dolmans MM and Donnez J: Potential involvement of hemoglobin and heme in the pathogenesis of peritoneal endometriosis. Fertil Steril 77: 561-570, 2002.

20. Nishie A, Ono M, Shono T, Fukushi J, Otsubo M, Onoue H, Ito Y, Inamura T, Ikezaki K, Fukui M, et al: Macrophage infiltration and heme oxygenase-1 expression correlate with angiogenesis in human gliomas. Clin Cancer Res 5: 1107-1113, 1999.

21. Hevir N, Ribič-Pucelj M and Lanišnik Rižner T: Disturbed balance between phase I and II metabolizing enzymes in ovarian endometriosis: A source of excessive hydroxy-estrogens and ROS? Mol Cell Endocrinol 367: 74-84, 2013.

22. Singh AK, Chattopadhyay R, Chakravarty B and Chaudhury K: Markers of oxidative stress in follicular fluid of women with endometriosis and tubal infertility undergoing IVF. Reprod Toxicol 42: 116-124, 2013. 
23. Sova H, Kangas J, Puistola U, Santala M, Liakka A and Karihtala P: Down-regulation of 8-hydroxydeoxyguanosine and peroxiredoxin II in the pathogenesis of endometriosis-associated ovarian cancer. Anticancer Res 32: 3037-3044, 2012.

24. Nourazarian AR, Kangari P and Salmaninejad A: Roles of oxidative stress in the development and progression of breast cancer. Asian Pac J Cancer Prev 15: 4745-4751, 2014.

25. Nitti M, Piras S, Marinari UM, Moretta L, Pronzato MA and Furfaro AL: HO-1 induction in cancer progression: A matter of cell adaptation. Antioxidants (Basel) 6: pii: E29, 2017.

26. Skrzypek K, Tertil M, Golda S, Ciesla M, Weglarczyk K, Collet G, Guichard A, Kozakowska M, Boczkowski J, Was $\mathrm{H}$, et al: Interplay between heme oxygenase-1 and miR-378 affects non-small cell lung carcinoma growth, vascularization, and metastasis. Antioxid Redox Signal 19: 644-660, 2013.
27. De Palma G, Mozzoni P, Acampa O, Internullo E, Carbognani P, Rusca M, Goldoni M, Corradi M, Tiseo M, Apostoli P and Mutti A: Expression levels of some antioxidant and epidermal growth factor receptor genes in patients with early-stage non-small cell lung cancer. J Nucleic Acids 2010: pii: 147528, 2010.

28. Kong B, Qia C, Erkan M, Kleeff J and Michalski CW: Overview on how oncogenic Kras promotes pancreatic carcinogenesis by inducing low intracellular ROS levels. Front Physiol 4: 246, 2013. 